\title{
Giant circular dichroism in extrinsic chiral metamaterials excited by off-normal incident laser beams
}

\author{
C. Feng a,b,*, Z.B. Wang ${ }^{\text {a,c,* }}$, S. Lee ${ }^{\text {a }}$, J. Jiao ${ }^{\text {a }}$, L. Li $^{\text {a }}$ \\ a School of Mechanical, Aerospace and Civil Engineering, the University of Manchester, Manchester, M13 9PL, UK \\ b Department of Chemistry, University of Leicester, University Road, Leicester, LE1 7RH, UK \\ c School of Electronics, Bangor University, Dean Street, Bangor, Gwynedd, LL57 1UT, UK
}

\section{A R T I C L E I N F O}

\section{Article history:}

Received 29 August 2011

Accepted 30 January 2012

Available online 11 February 2012

\section{Keywords:}

Metamaterial

Circular Dichroism

Chirality

Nanophotonics

\begin{abstract}
A B S T R A C T
Recently it was shown by experiments that circular dichroism (CD) can be observed in the metamaterials of nonchiral structures when they were subjected to obliquely incident light (E. Plum, et al., Physical Review Letters 102, $113902,2009)$. By far, external chirality simulation was only done for a particle array embedded in a homogenous medium (V. Yannopapas, Optics Letters 34, 5, 2009); no attempt has been made on simulating and modelling of circular dichroism in layered metamaterials (e.g., thin film on substrate structure). In this paper, we present the simulation of CD effect in layered external chiral metamaterials using CST software, theoretically investigate this intriguing phenomenon through a frequency domain finite integration technique, and optimize the metamaterial unit cell configurations (size, periodicity and film thickness) to maximize the $\mathrm{CD}$ phenomenon in near-infrared spectrum range. We show that the $\mathrm{CD}$ effect can be enhanced by five times using an optimized unit cell configuration, which is more than three times higher than the existing maximum theoretical results. The $\mathrm{CD}$ generation mechanism was elaborated with the help of induced surface current distributions.
\end{abstract}

(c) 2012 Elsevier B.V. All rights reserved.

\section{Introduction}

In 1968 V. G. Veslago introduced a theoretical concept of left handed material (LHM), also known as negative index medium (NIM) [1]. The first experimental prototype of LHM was demonstrated by D. R. Smith in 2000 [2]. At the same time, J. Pendry theoretically proposed that a slab of LHM could be used as 'perfect lenses' for perfect imaging [3]. These investigations set milestones on metamaterial research and many unique and intriguing properties of artificially engineered materials have been reported since then. One of the unique properties is the circular dichroism (CD) in metamaterials. CD effect, which was first introduced in 1997 [4], is the transmission intensity difference between the right-handed circularly polarized (RCP) light and the lefthanded circularly polarized (LCP) light. Due to its wide applications in photonic crystals, quantum devices, high density data storage [5] as well as in analytical chemistry, crystallography and molecular biology [6], CD effect in metamaterials has attracted increasing attentions in recent years.

The CD effect in metamaterials was initially observed in the intrinsic chiral structures only [7-11]. Soon it was discovered that CD phenomenon can also be observed in the metamaterials of non-chiral structures with an oblique incident laser beam, which was termed as 'extrinsic chiral' structures by the researchers at Southampton,

\footnotetext{
* Corresponding authors.

E-mail addresses: c.feng1016@gmail.com (C. Feng), zengbo.wang@gmail.com (Z.B. Wang).
}

UK [6]. The detected CD effect in extrinsic chiral structures was indistinguishable from that occurred in intrinsic chiral structure [6]. Compared to intrinsic chiral metamaterials, external chiral metamaterials are easier to design and fabricate, but more difficult to simulate because of the involvement of angled incident beams [6,12-14]. This paper, for the first time, demonstrates the simulation of $\mathrm{CD}$ effect in such layered external chiral metamaterials using a frequencydomain finite integration technology (FIT), as well as the optimization of the unit cell design configuration to maximize the $\mathrm{CD}$ effect within the near-infrared spectrum.

\section{Simulation methodology}

If we use "+" subscript for RCP light and "-" for LCP light, the following matrix equation can be used to correlate the transmission light $(T)$ and incident light $(I)$ :

$$
\left(\begin{array}{l}
T_{+} \\
T_{-}
\end{array}\right)=\left(\begin{array}{ll}
t_{++} & t_{-+} \\
t_{+-} & t_{--}
\end{array}\right)\left(\begin{array}{l}
I_{+} \\
I_{-}
\end{array}\right)
$$

The $2 \times 2 t$-matrix characterizes the transmission and conversion processes of RCP and LCP incident beams travelling through the metamaterial structures. While $t_{++}$representing the transmission amplitude of RCP incident light $I_{+}$and $t_{--}$representing the transmission amplitude of LCP incident light $I_{-}, t_{+-}$and $t_{-+}$represent the mutual conversion of RCP and LCP induced by the metamaterials and are the 
measure of circular birefringence. Mathematically CD is defined based on $t_{++}$and $t_{--}$as [6]:

$C D=\left|t_{++}\right|^{2}-\left|t_{--}\right|^{2}$

Because of the periodical boundary conditions set in our simulation, it is difficult to deal with directly using normal finite element method (FEM), our simulation is based on a frequency-domain FIT program CST software using unit cell boundary coupled with Floquet port. FIT is mathematical method to solve Maxwell Equation in the integral form, which can help solve the curve boundary directly other than approximated by stair effect as other methods. It is suitable for the problems ranging from static field calculations to high frequency applications in time or frequency domain [15]. The simulated structure is consisted of periodical arrays of identical unit cells and the incident beam is selected as the uniform plane wave. The oblique incidence condition was taken into account by a phase-shifted periodic boundary setting. Fig. 1 illustrates the experimental unit cell structure (Fig. $1 \mathrm{a}$ ) and dimension (Fig. 1b) used by the researchers who first discovered the external chirality in non-chiral metamaterials [6]. We will first simulate the $\mathrm{CD}$ effects of this unit cell metamaterial to compare with experiments, followed by further optimization to maximize the $C D$ effect. The unit cell consists of 50-nm-thick aluminium split nano-rings manufactured by e-beam lithography on a 1-mm-thick glass substrate. The period of the unit cell is $500 \mathrm{~nm}$. The optical properties of glass substrate and aluminium nanostructures used in modelling, which are varying with the incident beam frequencies, were taken from Ref. [16]. The thickness of glass substrates was set as $1500 \mathrm{~nm}$ against the real value $1-\mathrm{mm}$ in all simulations, following a series of tests which show that thicker substrate $(t>1500 \mathrm{~nm}$ ) play negligible influence on the CD effect of studied metamaterial.

\section{Results and discussion}

\subsection{Comparison of simulated $C D$ effect with experiments}

Fig. 2 shows the comparison of the $\mathrm{CD}$ effect between the simulated and the corresponding experimental results of the above metamaterial structure between $800 \mathrm{~nm}$ and $2000 \mathrm{~nm}$ wavelengths in near-IR range. The red and blue points plotted are the experimental result collected by the researchers from Southampton at the $-20^{\circ}$ and $+20^{\circ}$ incident respectively, while the curves are the simulated $\mathrm{CD}$ effect at the same conditions.

As it can be seen, the simulated CD curve has irregular sharp peaks (Peak A) in region A (wavelength $<1000 \mathrm{~nm}$ ), which are caused by optical diffraction. Diffraction occurs when the light wavelength is

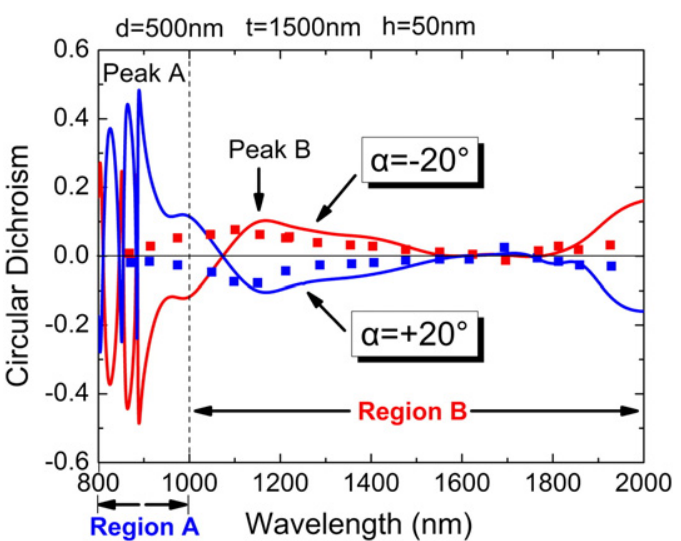

Fig. 2. Comparison the $\mathrm{CD}$ spectrum of extrinsic chiral metamaterial between the simulated result (the curves) and the corresponding experimental result (the discrete points) from ref [6].

analogous with the unit cell period so that the transmitted light from adjacent unit cell can interfere with each other. Theoretically when the wavelength of the incident light is about two times over the unit cell period, diffraction effect becomes weak enough to be neglected, and the medium can be treated as homogenous media [17]. In the range of wavelength over $1000 \mathrm{~nm}$ (region B), CD spectrum shown in our simulation varies smoothly; the tendency and the peak amplitude were both similar to the experimental results. The reason why diffraction peaks were not presented in the experimental results is possibly due to the insufficient points collected during experiments within the $\mathrm{CD}$ spectrum; only two experimental points was collected for wavelengths below $900 \mathrm{~nm}$, which is not possible to reflect possible oscillation caused by diffraction in this region. The main CD peak observed by experiments at wavelength $1100 \mathrm{~nm}$ with $\mathrm{CD}=0.075$ was in very good agreement with simulation (Peak B in Fig. 2: wavelength $=1150 \mathrm{~nm}, C D=0.1$ ). Despite of slight difference between theory and experiment for long wavelengths $>1800 \mathrm{~nm}$, which could again be caused by insufficient data sampling in experiments, the simulation result curve in region B $(1100 \mathrm{~nm}<$ wavelength $<2000 \mathrm{~nm}$ ) matches very well with experiments. Taking the experimental limit on insufficient sampling points into account, it is reasonable to conclude that our simulation agrees well with experiments.

We now turn to study the influence of other unit cell conditions such as different film thickness and pattern size, aiming to understand the key factors affecting $\mathrm{CD}$ and consequently optimize the $\mathrm{CD}$ effect.

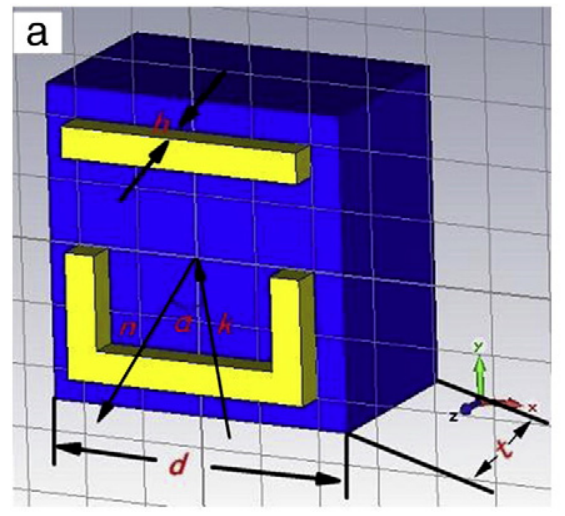

b

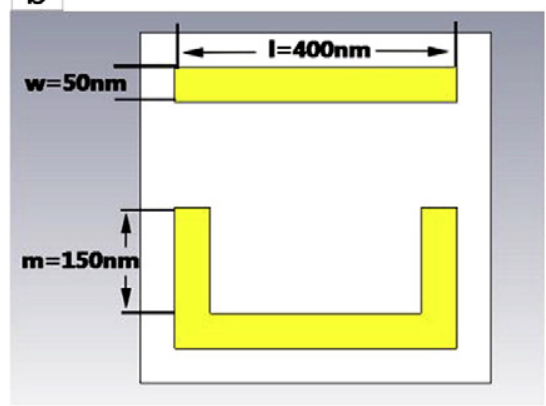

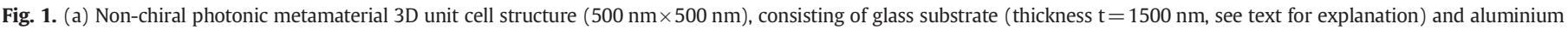

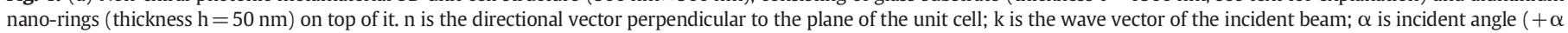
in drawing). (b) Dimensions of the aluminium pattern. 


\section{2. $C D$ effect with varying the unit cell conditions}

The effects of the metallic pattern scales and the film thickness on the $\mathrm{CD}$ phenomenon were investigated, shown in Fig. 3. The scaling was only for metallic pattern while the unit cell size was fixed at $500 \mathrm{~nm}$. For a clear description, we neglected the high oscillation diffraction peaks (Peak A) and set the starting spectrum wavelength at $900 \mathrm{~nm}$ in this section. For simplicity, we only show the $-20^{\circ}$ incident result in each case, following the fact that the $\mathrm{CD}$ spectrum for $\alpha=+20^{\circ}$ is nearly symmetric to that for $\alpha=-20^{\circ}$.

As it can be seen, shrinking the size of metallic pattern can greatly affect the $\mathrm{CD}$ spectrum of studied metamaterial: When the pattern size is scaled down to a quarter of its original size, the $C D$ effect was eliminated completely for wavelengths $>1000 \mathrm{~nm}$ due to negligible response from metallic patterns at these wavelengths. However, pattern shrinking to a half will not weaken $C D$ peak amplitude, while the wavelength in terms of max peak was blue-shifted from $1150 \mathrm{~nm}$ to $1280 \mathrm{~nm}$.

When changing the metallic film thickness, the exhibiting $C D$ phenomenon became interesting. On one hand, as the film thickness reduced, the Peak B shrank greatly, however on the other hand, another striking peak called Peak $C$ emerged when $\mathrm{h}=10 \mathrm{~nm}$ and $\mathrm{h}=5 \mathrm{~nm}$. The amplitude of Peak C was much higher than Peak B, which can also be used for characterising the $\mathrm{CD}$ phenomenon. The results led us to believe that to obtain a remarkable $\mathrm{CD}$ phenomenon it is not necessary to fabricate thick metallic pattern on the substrate. Thinner metallic pattern film could be a better choice with higher CD amplitudes.

\subsection{The optimum design configuration of the unit cell and its maximum CD spectrum}

The discussion above gives an indication that the maximum $C D$ spectrum emerges around $\mathrm{h}=10 \mathrm{~nm}$. After a series of simulation
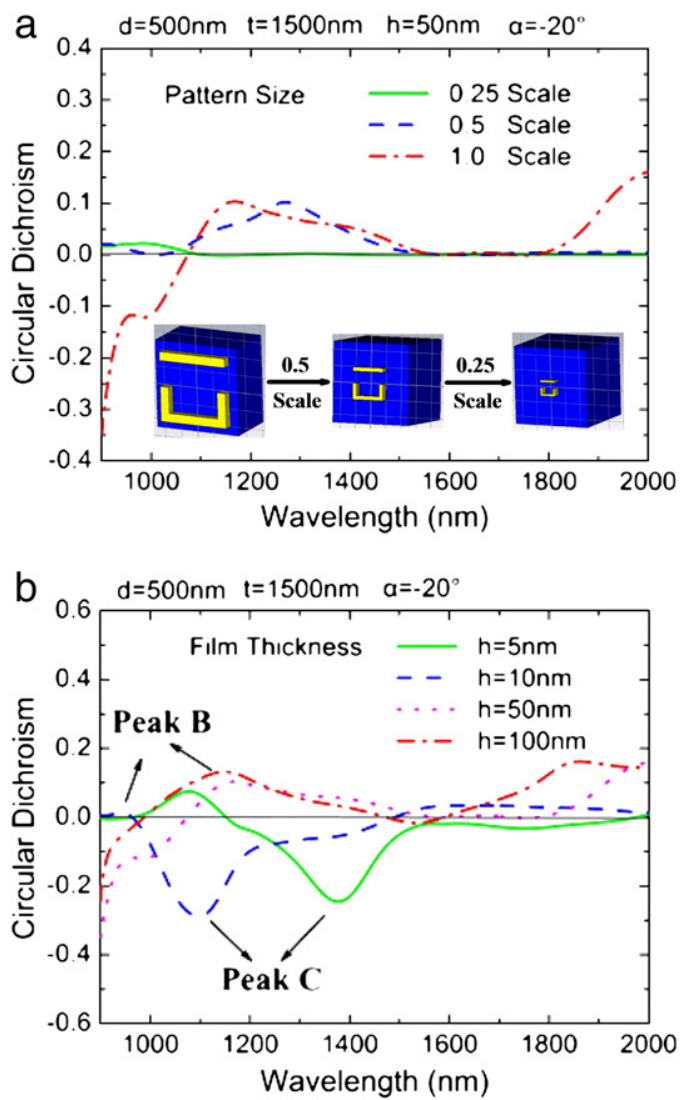

Fig. 3. a) Dependence of $C D$ effect on metallic pattern size with an area scale factor of $1,0.5$ and 0.25 . b) Dependence of CD effect on film thickness varying from $5 \mathrm{~nm}$ to $100 \mathrm{~nm}$. tests, it was confirmed that $\mathrm{CD}$ amplitude reaches its maximum at $\mathrm{h}=15 \mathrm{~nm}$ when fixing other parameters at $\mathrm{t}=1500 \mathrm{~nm}, \mathrm{~d}=500 \mathrm{~nm}$. This is the optimum settings of the unit cell structure. The optimized CD spectrum is shown in Fig. 4.

It has been found that in the optimum setting of the unit cell, the diffraction amplitude was controlled below 0.1 and its dominant region was confined below $900 \mathrm{~nm}$. The peak of the CD spectrum reached about 0.365 at around $1125 \mathrm{~nm}$ wavelength. This peak value was about 5 times higher than the experimental results obtained by the previous researchers [6], and also almost 4 times higher than the initial simulation results when $h=50 \mathrm{~nm}$, as shown in Fig. 2. If compared with the $\mathrm{CD}$ effect exhibited in the particle arrays, whose maximum amplitude was calculated of about 0.11 [12], it can be found that the result here shows a much more prominent CD effect.

\subsection{Surface current distributions at the peak wavelength}

To study the fundamental principles of the $\mathrm{CD}$ effect generated by oblique incident beam on non-chiral metamaterials, the instantaneous near-field distributions of the electric field and surface current at the peak wavelength $=1028 \mathrm{~nm}$ and $-20^{\circ}$ incident as in Fig. 4 were calculated and shown in Fig. 5(a) and (b). It shows the current distributions when phase $=0^{\circ}$, and they change their flowing direction every $90^{\circ}$, thus the period is $180^{\circ}$. It can be seen that, the induced surface current, which was associated with the excitation of surface plasmons in metallic patterns, was only observed within the area of the metallic pattern. In general, surface plasmons will not be excited on glass substrate; under some special wavelength within the midIR range, however, glass can perform like metal with excited surface plasmons, which affects $C D$. This will be reported in a separate publication.

There exist significant differences between the RCP and LCP instances in term of the modes of surface current distributions. For the RCP incident light, the excited current derives from and vanishes at the end point (A, B, C and D) and the corner point ( $E$ and $F$ ) of the metallic pattern, however for the LCP light, it can also generate and counteract at the middle of the nether bar (Point $G$ ).

\subsection{Mechanism of $C D$ effect in near-IR range}

In previous literatures, the mechanism of external chirality was thought relating to optical activity: the retarded wave caused by the magnetic dipole moment rotating the polarization state of the transmitted light $[6,13,14]$. Here, we use surface current distributions results shown in Fig. 5 to further clarify the CD generation mechanism in a more detailed and precise manner.

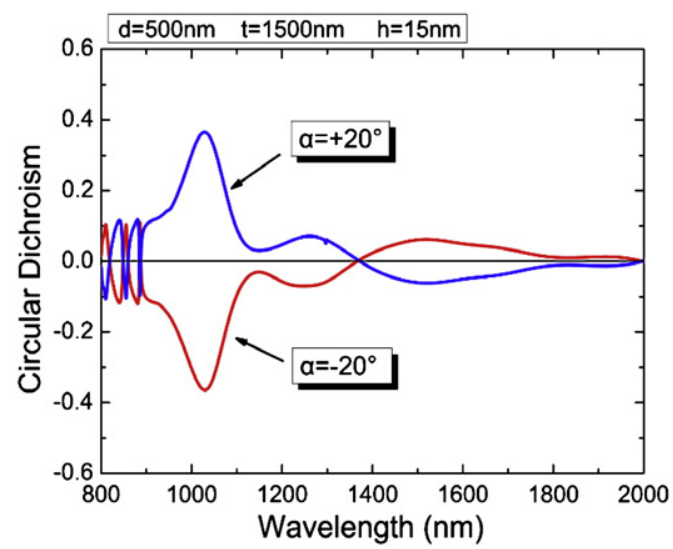

Fig. 4. Optimized $C D$ spectrum with maximum peak value $C D=0.365$ at $1028 \mathrm{~nm}$ wavelength, under conditions of $d=500 \mathrm{~nm}, \mathrm{t}=1500 \mathrm{~nm}, \mathrm{~h}=15 \mathrm{~nm}$. 


\section{Surface Current Distribution}
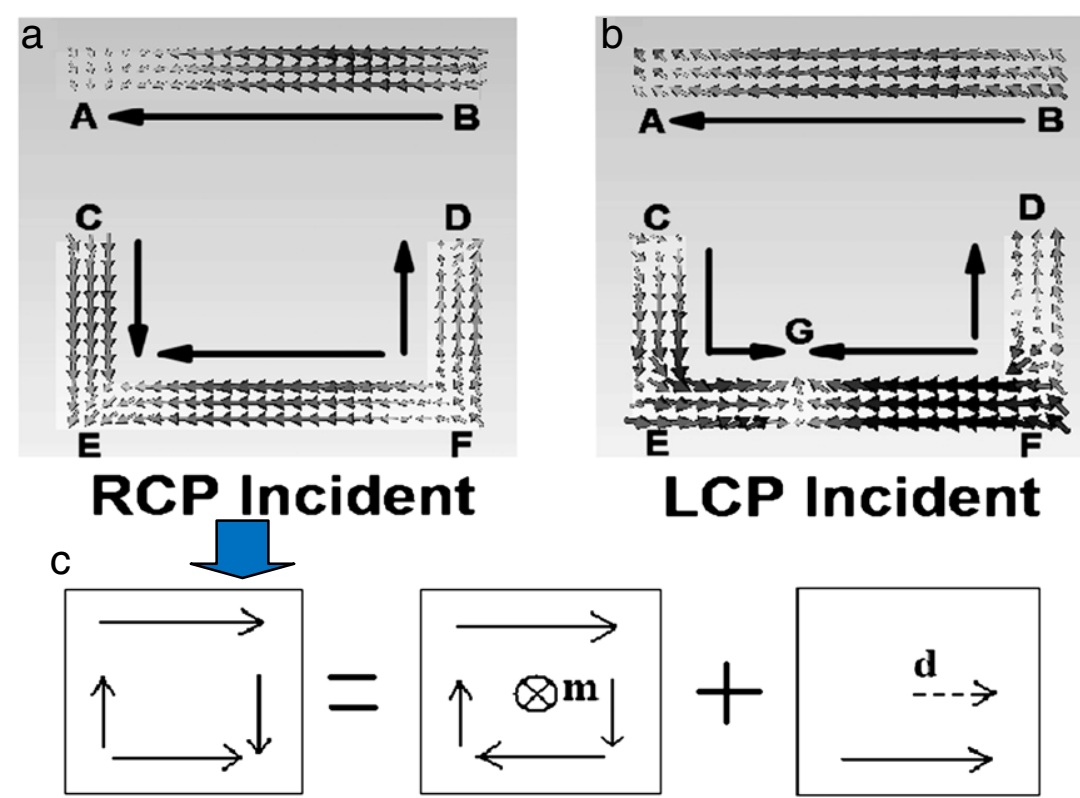
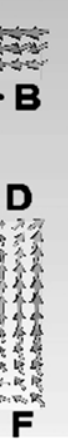

t

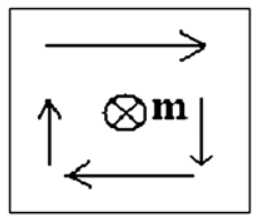

(Symmetric Current)

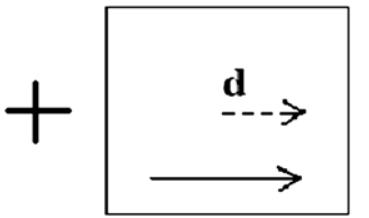

(Anti-symmetric Current)

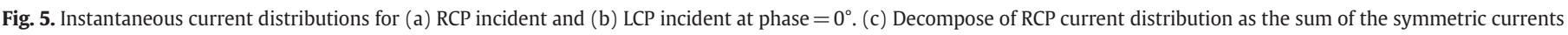
(exciting magnetic dipole moment) and anti-symmetric current (exciting electric dipole moment).

In the case of RCP light incident, the excited surface current distributions can be regarded as the sum of the symmetric and antisymmetric currents which induce electric dipole moment, $d$, and the magnetic dipole moment, m, respectively, as shown in Fig. 5 (c). When the light was at oblique incident, the moment $\mathrm{d}$ and $\mathrm{m}$ would have a projection component on the plane perpendicular to the light incident direction, marked as $\mathrm{d}^{\prime}$ and $\mathrm{m}^{\prime}$ respectively (Fig. 6). For RCP light, the polarization state, $\mathrm{E}$ and $\mathrm{H}$, both rotate towards the right hand side viewing from the incident direction, while from the visual angle in Fig. 6, it should rotate counter-clockwise. However, the projection moments $\mathrm{d}^{\prime}$ and $\mathrm{m}^{\prime}$ both create a tendency that rotates the both polarization states to the opposite direction. This resistance effect will weaken the RCP light transmission and will be throughout the transmission process because the periods of the excited surface current and the polarization rotation of the incident light are identical. Consequently, the remaining RCP light after transmission is always less than regular.

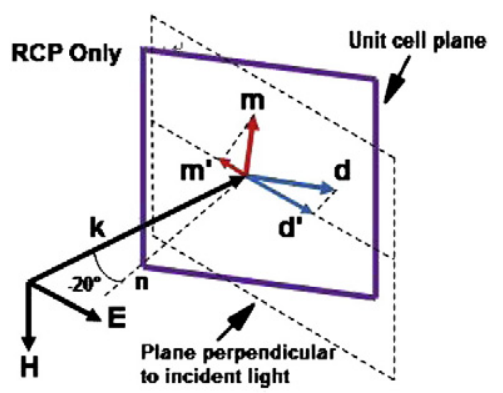

Fig. 6. Schematic representation of $C D$ generation mechanism for RCP incident: The projection component on the plane perpendicular to the incident light (the dash frame in the figure) of the magnetic dipole moment $\left(\mathrm{m}^{\prime}\right)$ and the electric dipole moment $\left(d^{\prime}\right)$ resist the polarization state of the incident light from rotating towards their natural direction, thus reduces the transmission intensity and results in CD.
On the other hand, because the excited surface currents of the LCP incident instance could not be treated as RCP, the resistance effect of the polarization rotation to the LCP light was not existed. So the LCP light transmitted as regular. As a result, a large difference in transmission intensity between the LCP and RCP light was generated, which is the circular dichroism effect. Thus, $C D$ peak at $-20^{\circ}$ incident in this case should be a large negative value, which is consistent with the simulation result shown in the Fig. 4. In summary, this hypothesis is fully consistent with both the simulation and experimental results, which can exactly explain the mechanism of $\mathrm{CD}$ generation.

\section{Conclusion}

Circular dichroism in layered non-chiral metamaterials induced by an oblique incident beam has been studied theoretically in this paper. The applied model was firstly verified by comparing simulation results with experimental results. The unit cell structure was then optimized, leading to a five times increase in the peak $C D$ value, from 0.075 to 0.365 , which is the maximum CD value obtained by simulation to date. The near-field and surface current distributions of this simulation study not only justified the assumed simple model to explain the optical activity, but also led to a more detailed and precise explanation of $\mathrm{CD}$ generation mechanism - the projection components of the electric and magnetic moments induced by the excited surface current tends to prevent one of the polarization states of the incident light from rotating as in the usual way, and the large resistance difference between the LCP and RCP light is the essential reason of $\mathrm{CD}$ generation.

\section{References}

[1] V.G. Veselago, Soviet Physics Uspekhi 10 (1968) 509.

[2] D.R. Smith, W.J. Padilla, D.C. Vier, S.C. Nemat-Nasser, S. Schultz, Physical Review Letters 84 (2000) 4184.

[3] J.B. Pendry, Physical Review Letters 85 (2000) 3966.

[4] A. Sochava, C. Simovski, S. Tretyakov, "Chiral effects and eigenwaves in bi-anisotropic 263 omega structures" in "Advances in Complex Electromagnetic Materials" [book 
auth.] A. Priou, A. Sihvola, S. Tretyakov, A. Vinogradov, Dordrecht-Boston-London: Kluwer Academic Publishers, pp. 85-102, 1997.

[5] Q. Xie, M.H. Hong, H.L. Tan, G.X. Chen, L.P. Shi, T.C. Chong, Journal of Alloys and Compounds (2008) 261.

[6] E. Plum, X.X. Liu, V.A. Fedotov, Y. Chen, D.P. Tsai, N.I. Zheludev, Physical Review Letters 102 (2009) 113902.

[7] M. Decker, M.W. Klein, M. Wegener, S. Linden, Optics Letters 32 (2007) 856.

[8] E. Plum, J. Zhou, J. Dong, V.A. Fedotov, T. Koschny, C.M. Soukoulis, N.I. Zheludev, Physical Review B 79 (2009) 035407.

[9] E. Plum, V.A. Fedotov, A.S. Schwanecke, N.I. Zheludev, Applied Physics Letters 90 (2007) 223113.

[10] J.K. Gansel, M. Thiel, M.S. Rill, M. Decker, K. Bade, S. Volker, G.V. Freymann, S. Linden, M. Wegener, Science 325 (2009) 1513.
[11] B. Wang, J. Zhou, T. Koschny, C.M. Soukoulis, Applied Physics Letters 94 (2009) 151112.

[12] V. Yannopapas, Optics Letters 34 (2009) 5.

[13] E. Plum, V.A. Fedotov, N.I. Zheludev, Applied Physics Letters 93 (2008) 191911.

[14] E. Plum, V.A. Fedotov, N.I. Zheludev, Journal of the Optical Society of America. A 11 (2009) 074009.

[15] T. Weiland, Electronics and Communication 31 (1977) 116.

[16] E.D. Palik, Handbook of Optical Constants of Solids, 3rd ed. Academic, New York 1985.

[17] W. Cai, V. Shalaev, Optical Metamaterials: Fundamentals and Applications, Springer Science+Business Media, LLC, 2010. 